\title{
THE EXPERIENCE AND RESULTS OF CYTOREDUCTIVE SURGERY AND HIPEC USED IN ADVANCED OVARIAN CANCER
}

\author{
Oleksandr Bondar ${ }^{1}$, Andriy Rybin ${ }^{2}$ \\ ${ }^{1}$ Department of X-ray diagnostics, radiotherapy and radiation medicine with a course of oncology, Odesa National Medical University, \\ Odessa, Ukraine \\ ORCID: http://orcid.org/0000-0001-8746-1878 \\ ovbondar0708@gmail.com \\ ${ }^{2}$ Department of X-ray diagnostics, radiotherapy and radiation medicine with a course of oncology, Odesa National Medical University, \\ Odessa, Ukraine \\ ORCID: http://orcid.org/0000-0002-1145-6690
}

A RTICLE INFO
Article history:
Received date 18.09 .2020
Accepted date 22.10 .2020
Published date 30.10 .2020
Section:
Practical medicine
DO I

$10.21303 / 2313-8416.2020 .001458$

KEYWORDS

ovarian cancer

treatment

HIPEC

cytoreduction

chemotherapy

\section{ABSTRACT}

The research object was life quality of patients with ovarian cancer after complex treatment. Nowadays cytoreductive surgery and HIPEC is the mainstay of management of advanced stages ovarian cancer.

Description of the problem: study was conducted to assess the effectiveness of combined treatment in IIIA-IIIC ovarian cancer, its impact on quality of life.

Main scientific results: 37 patients of main group (CRS + HIPEC) were compared with 25 patients of control group (surgery + systemic chemotherapy). The quality of life was assessed with Medical Outcomes Study 36-Item Short Form Health Survey (SF-36). Comparative analysis of quality of life 6 months after treatment completion did not show significant statistical difference. Combination of cytoreduction with HIPEC improves quality of life in patients with ovarian cancer, is tolerated better and has less systemic toxicities than systemic chemotherapy.

The area of practical use of research results: hyperthermic intraperitoneal chemotherapy is performed for patients suffering from malignant tumors of the abdominal cavity, including metastatic colon cancer, ovarian cancer, stomach cancer, pseudomyxoma, mesothelioma, rhabdomyosarcoma, and some other types of oncology.

Innovative technological product: combination of cytoreduction with HIPEC improves quality of life in patients with ovarian cancer.

Scope of application of an innovative technological product: HIPEC is an innovative treatment option for advanced cancer that has spread to the abdominal cavity (carcinomatosis) and has not gone beyond it.

(C) The Author(s) 2020. This is an open access article under the CC BY license http://creativecommons.org/licenses/by/4.0).

\section{Introduction}

\section{1. The research object}

Life quality of patients with ovarian cancer after complex treatment

\section{2. Description of the problem.}

Ovarian cancer remains a complicated medical issue. According to worldwide statistics, 1-year, 3-year and 5-year survival rates are $63 \%, 41 \%, 35 \%$, respectively [1]. In the last decade a modest decrease of 5-year survival was caused by more common use of platinum-based chemotherapy for disseminated ovarian cancer [2].

More than $70 \%$ cases of ovarian cancer are revealed at late stages, that accounts for poor prognosis. Contemporary treatment standards include combination of surgical cytoreduction and platinum-based chemotherapy. However, even after complete cytoreduction with adjuvant first-line systemic treatment, that achieved complete clinical regression, 5-year survival rates for III and IV stages are $20 \%$ and $10 \%$, respectively [3].

\section{3. Suggested way to solve the problem}

Most patients respond well to first-line therapy, although $30 \%$ may have a platinum-resistant or platinum-refractory tumors. In such cases other cytostatic drug combinations, target thera- 
py and immunotherapy is recommended [4]. To sum up, despite absence of clinical signs, one third of patients after first-line chemotherapy will have relapse in 2-3 years. Objective response rate is $10-25 \%$, median survival time is $7-18$ months [5]. Most studies share an opinion, that optimal cytoreduction is crucial for effective treatment. However, only $10-15 \%$ of performed surgeries achieve optimal extent. That is why it is still relevant to improve methods of surgical and adjuvant methods of ovarian cancer treatment [6].

The aim of the research. The study was conducted to assess the effectiveness of cytoreductive surgery in combination with hyperthermic intraperitoneal chemoperfusion (HIPEC) in advanced stages of ovarian cancer management. The article displays the experience of University clinic of Odessa National Medical University.

\section{Materials and methods}

37 cases of ovarian cancer treated with cytoreduction, HIPEC and 4-6 courses of adjuvant chemotherapy were analyzed. Study has been conducted in the Department of Surgery No. 4 with the Course of Oncology (Odessa National Medical University) since 2016 and is continued now. All patients had serosal papillary ovarian cancer FIGO IIIA-IIIC. Patients age was 31-59 years, the middle age was $43 \pm 7$ years old. Control group included 25 patients after surgical treatment and convenient systemic chemotherapy. All patients received suboptimal cytoreduction.

Before including patients in the study protocol, personal written voluntary consents were obtained to participate in the study in accordance with the Helsinki Declaration of the World Medical Association (protocol No. 119A bioethics commission meetings of Odessa National Medical University, 15.05.2016)

Criteria of exclusion were age more than 70 years, extensive canceromatosis with peritoneal canceromatosis index more than 20 , unresectable small bowel involvement, distant metastasis, retroperitoneal lymphadenopathy, unresectable retroperitoneum invasion, severe concomitant pathology. Previous surgeries with massive adhesions were considered as relative contraindication.

Cytoreduction included visceral resections and peritonectomy. Hysterectomy with bilateral adnexectomy, omentectomy and selective parietal peritonectomy was performed.

HIPEC is performed in the University clinic of Odessa National Medical University with Performer LRT (Rand, Italy). Its main components are two pumps, heater, infusion lines and digital integrational system. The apparatus controls the procedure automatically and allows fine tuning and monitoring of different parameters like temperature, volume speed, target volumes and timing.

All patients in the main group received HIPEC with cisplatine and doxorubicine. Drugs are dissolved in 5000-6000 $\mathrm{ml}$ of isotonic perfusate. Closed technique was used, that is after wound closure 5-6 silicone drains are placed into abdominal and pelvic cavity. Procedures lasted $60-90$ minutes with target intraperitoneal temperature $40-41{ }^{\circ} \mathrm{C}$ and volume speed $800-900 \mathrm{ml} / \mathrm{min}$. Mean filling volume is $2500-3000 \mathrm{ml}$ depending of patients constitution. The washout phase takes $10-15$ minutes until clear outflow and normothermia is achieved.

All patients received cytoprotection with thiosulfate during HIPEC and 6 hours after to prevent systemic toxicity of cisplatine. Usually patients stayed for one day in the intensive care unit. Perioperative medications include dexamethasone, 5-HT blockers, adequate analgesics and infusional therapy. Antibiotic prophylaxis was protracted for 1-3 days if needed.

Patients were assessed intraoperatively according to peritoneal canceromatosis index, previous surgical score, cytoreduction completeness score. The quality of life was analyzed with Medical Outcomes Study 36-Item Short Form Health Survey (SF-36). Adverse effects and toxicities were secondary outcomes of the study. Time series statistics were used to analyze the obtained data. To assess the significance of these changes, was used the Mann-Whitney parametric test. SPSS Statistics was used as software for estimating statistics.

\section{Results}

Among the main group ( $\mathrm{n}=37)$ IIIA, IIIB, IIIC stages were revealed in 5, 3 and 29 patients, respectively. In the control group ( $\mathrm{n}=25), 10$ patients had IIIA, 2 patients - IIIB, 13 patients - IIIC. Canceromatosis index in the main group was LS-1 (28.0\%) and LS-2 (72.0\%). It did not differ significantly in control group: LS- $1-36.0 \%$, LS- $2-64.0 \%$. Cytoreduction completeness score had no statistical difference (CC-1 and CC-2 was $28.0 \%, 72.0 \%$ in main group, $36.0 \%, 64.0 \%$ in control group). 
Comparative analysis of quality of life 6 months after treatment completion did not showed nonsignificant statistical difference (Table 1).

Table 1

Comparison of the Short Form (36) Health Survey

\begin{tabular}{ccc}
\hline SF-36 Scale & Main group $(\mathbf{n}=\mathbf{3 7})$ & Control group $(\mathbf{n}=\mathbf{2 5})$ \\
\hline Physical activity & $34.08 \pm 21.14$ & $31.88 \pm 19.44$ \\
Physical role functioning & $28.42 \pm 13.43$ & $21.01 \pm 14.23$ \\
Bodily pain & $46.29 \pm 16.23$ & $41.27 \pm 20.11$ \\
General health perceptions & $35.33 \pm 14.76$ & $33.72 \pm 23.27$ \\
Vitality & $49.85 \pm 24.16$ & $44.25 \pm 34.26$ \\
Social role functioning & $65.42 \pm 26.16$ & $63.12 \pm 22.39$ \\
Emotional role functioning & $42.11 \pm 15.46$ & $44.25 \pm 17.24$ \\
Mental health & $66.32 \pm 22.26$ & $59.32 \pm 22.26$
\end{tabular}

No allergic and idiosyncratic drug reactions were observed in the main group. 9 patients had temporary hyperthermic reaction, controlled with NSAIDs.

Few patients $(n=5,13.5 \%)$ complained about pain around drain contrapertures.

Toxicities comparison of treatment regimens is shown in the Tables 2, 3 .

Table 2

Adverse effects of treatment in the main group

\begin{tabular}{|c|c|c|c|c|c|}
\hline \multirow{2}{*}{ Toxicity } & \multicolumn{4}{|c|}{ Toxicity grade } & \multirow{2}{*}{ Overall, \% } \\
\hline & I, n (\%) & II, n (\%) & III, n (\%) & IV, n (\%) & \\
\hline Leukopenia & $13(35.1)$ & $7(18.8)$ & $4(10.8)$ & $3(8.1)$ & 72.8 \\
\hline Neutropenia & $12(32.4)$ & $6(16.2)$ & $5(13.5)$ & - & 62.1 \\
\hline Anemia & $3(8.1)$ & $2(5.4)$ & - & - & 13.5 \\
\hline Thrombocytopenia & $6(16.2)$ & - & - & - & 16.2 \\
\hline Nausea & $7(18.9)$ & $5(13.5)$ & $5(13.5)$ & - & 45.9 \\
\hline Vomiting & $16(43.2)$ & $1(2.7)$ & $1(2.7)$ & - & 48.6 \\
\hline Diarrhea & $3(8.1)$ & - & - & - & 8.1 \\
\hline Stomatitis & $8(21.6)$ & $1(2.7)$ & - & - & 24.3 \\
\hline
\end{tabular}

Table 3

Adverse effects of treatment in the control group

\begin{tabular}{|c|c|c|c|c|c|}
\hline \multirow{2}{*}{ Toxicity } & \multicolumn{4}{|c|}{ Toxicity grade } & \multirow{2}{*}{ Overall, \% } \\
\hline & I, n (\%) & II, n (\%) & III, n (\%) & IV, n (\%) & \\
\hline Leukopenia & $10(40.0)$ & $8(32.0)$ & $1(4.0)$ & $1(4.0)$ & 80.0 \\
\hline Neutropenia & $11(44.0)$ & $4(16.0)$ & $2(8.0)$ & $1(4.0)$ & 72.0 \\
\hline Anemia & $5(20.0)$ & $4(16.0)$ & $4(16.0)$ & - & 52.0 \\
\hline Thrombocytopenia & $4(16.0)$ & - & - & - & 8.0 \\
\hline Nausea & $7(28.0)$ & $4(16.0)$ & $4(16.0)$ & - & 60.0 \\
\hline Vomiting & $10(40.0)$ & $4(16.0)$ & $1(4.0)$ & - & 60.0 \\
\hline Diarrhea & $3(12.0)$ & - & - & - & 12.0 \\
\hline Stomatitis & $2(8.0)$ & $4(16.0)$ & $1(4.0)$ & - & 28.0 \\
\hline
\end{tabular}


Hematological, gastrointestinal adverse effects were comparable in both groups. Less nephrotoxicity was observed in the control group.

Mean hospital stay was 8.9 days in the main group and did not differ significantly from control group.

\section{Discussion}

Cytoreduction and HIPEC have a reasonable rationale. Several tumors (ovarian cancer, malignant mesothelioma, colorectal and gastric cancer), that evolve into peritoneal carcinomatosis, in some stages of development are locally contained within serosal lining without giving distant metastases [7, 8]. Macroscopic tumor removal with surgical cytoreduction and microscopic with HIPEC achieves locoregional control.

By definition, chemotherapeutic drugs have various grades of systemic toxicities. Their use in high concentrations may cause serious adverse effects. Regional chemotherapy can achieve high local concentrations without systemic leakage into systemic circulation. Hyperthermia has direct selective cytotoxicity and acts synergistically with alkylating agents, like anthracyclines [9, 10]. In addition, it improves drug penetration into tumor deposits. Continuous cavity chemoperfusion supports constant chemotherapeutic agents concentration and equal distribution. These features explain better "local" intraperitoneal control after HIPEC comparing to systemic chemotherapy.

Study limitations. The study was carried out on the basis of one clinic, the study was not placebo-controlled, the patients were not divided into groups according to BRCA.

The research is continued now and the new results will be published as soon as possible.

\section{Conclusions}

1. Combination of cytoreduction with HIPEC provides significant prolongation of life for patients with advanced ovarium cancer and increases their life quality.

2. HIPEC is proven to be effective in conjunction with optimal or suboptimal cytoreduction.

3. HIPEC is tolerated better and has less systemic toxicities in comparison with systemic chemotherapy. Minimizes harm to other organs outside the abdominal cavity. Reduces some of the side effects of systemic chemotherapy.

4. Unlike systemic chemotherapy, which enters the body through the circulatory system and circulates throughout the body, in the HIPEC procedure, the chemotherapy drug acts directly on cancer cells that have spread in the abdominal cavity. This allows for high-dose chemotherapy treatments. Heating the chemotherapeutic method allows to improve the degree of absorption of drugs by tumor cells and to achieve an effect on malignant cells remaining in the abdominal cavity after surgical resection of visible tumor foci.

\section{Conflicts of interest}

The authors declare that they have no conflicts of interest.

\section{References}

[1] Bulletin of National Cancer Registry of Ukraine (2019). Kyiv, 21. Available at: http://www.ncru.inf.ua/publications/BULL_21/ index_e.htm

[2] Global Cancer Observatory. Available at: https://gco.iarc.fr/

[3] Cotte, E., Glehen, O., Mohamed, F., Lamy, F., Falandry, C., Golfier, F., Gilly, F. N. (2007). Cytoreductive Surgery and Intraperitoneal Chemohyperthermia for Chemoresistant and Recurrent Advanced Epithelial Ovarian Cancer: Prospective Study of 81 Patients. World Journal of Surgery, 31 (9), 1813-1820. doi: http://doi.org/10.1007/s00268-0079146-8

[4] Parson, E. N., Lentz, S., Russell, G., Shen, P., Levine, E. A., Stewart, J. H. (2011). Outcomes after cytoreductive surgery and hyperthermic intraperitoneal chemotherapy for peritoneal surface dissemination from ovarian neoplasms. The American Journal of Surgery, 202 (4), 481-486. doi: http://doi.org/10.1016/j.amjsurg.2011.02.004

[5] Di Giorgio, A., Naticchioni, E., Biacchi, D., Sibio, S., Accarpio, F., Rocco, M. et. al. (2008). Cytoreductive surgery (peritonectomy procedures) combined with hyperthermic intraperitoneal chemotherapy (HIPEC) in the treatment of diffuse peritoneal carcinomatosis from ovarian cancer. Cancer, 113 (2), 315-325. doi: http://doi.org/10.1002/cncr.23553 
[6] Bakrin, N., Classe, J. M., Pomel, C., Gouy, S., Chene, G., Glehen, O. (2014). Hyperthermic intraperitoneal chemotherapy (HIPEC) in ovarian cancer. Journal of Visceral Surgery, 151 (5), 347-353. doi: http://doi.org/10.1016/j.jviscsurg. 2014.07.005

[7] Onda, T., Kobayashi, H., Nakanishi, T., Hatae, M., Iwasaka, T., Konishi, I. et. al. (2009). Feasibility study of neoadjuvant chemotherapy followed by interval debulking surgery for stage III/IV ovarian, tubal, and peritoneal cancers: Japan Clinical Oncology Group Study JCOG0206. Gynecologic Oncology, 113 (1), 57-62. doi: http://doi.org/10.1016/ j.ygyno.2008.12.027

[8] Di Giorgio, A., Naticchioni, E., Biacchi, D., Sibio, S., Accarpio, F., Rocco, M. et. al. (2008). Cytoreductive surgery (peritonectomy procedures) combined with hyperthermic intraperitoneal chemotherapy (HIPEC) in the treatment of diffuse peritoneal carcinomatosis from ovarian cancer. Cancer, 113 (2), 315-325. doi: http://doi.org/10.1002/ cncr. 23553

[9] Chua, T. C., Yan, T. D., Saxena, A., Morris, D. L. (2019). Should the treatment of peritoneal carcinomatosis by cytoreductive surgery and hyperthermic intraperitoneal chemotherapy still be regarded as a highly morbid procedure? A systematic review of morbidity and mortality. Annals of Surgery, 249 (6), 900-907. doi: http://doi.org/10.1097/sla.0b013e318 1a45d86

[10] Bristow, R. E., Tomacruz, R. S., Armstrong, D. K., Trimble, E. L., Montz, F. J. (2002). Survival Effect of Maximal Cytoreductive Surgery for Advanced Ovarian Carcinoma During the Platinum Era: A Meta-Analysis. Journal of Clinical Oncology, 20 (5), 1248-1259. doi: http://doi.org/10.1200/jco.2002.20.5.1248 